\title{
CHARACTERIZATION OF HILBERT FUNCTIONS OF GORENSTEIN ARTIN ALGEBRAS WITH THE WEAK STANLEY PROPERTY
}

\author{
TADAHITO HARIMA
}

(Communicated by Eric Friedlander)

\begin{abstract}
We give a characterization of Hilbert functions of Gorenstein Artin algebras with the weak Stanley property. Namely, we prove that a necessary and sufficient condition for a given O-sequence $\underline{H}$ to be the Hilbert function of some Gorenstein Artin algebra with the weak Stanley property is that the sequence $\underline{H}$ is an SI-sequence.
\end{abstract}

\section{INTRODUCTION}

A standard G-algebra $A=A_{0} \oplus A_{1} \oplus \cdots$ over a field $k$ is a commutative graded ring such that $A_{0}=k, A$ is generated as a $k$-algebra by $A_{1}$ and the dimension of $A_{1}$ as a $k$-vector space is finite. Thus they are those $k$-algebras which can be written in the form $A=k\left[x_{0}, x_{1}, \ldots, x_{n}\right] / I$, where the $x_{i}$ are indeterminates of degree 1 and $I$ is a homogeneous ideal. In this paper a standard $G$-algebra over $k$ is called a $k$-algebra for short.

The Hilbert function of $A$ is defined by $H(A, i)=\operatorname{dim}_{k} A_{i}$ for all $i=$ $0,1, \ldots$, and $H(A, i)=0$ for all $i<0$. In particular, if $A$ is Artin, then we denote by the finite sequence $H(A)=(H(A, 0), H(A, 1), \ldots, H(A, s))$ the Hilbert function of $A$, where $s=\max \left\{i \mid A_{i} \neq 0\right\}$. Furthermore, we put $\sigma(A)=s+1$.

If $h$ and $i$ are positive integers, then $h$ can be written uniquely in the form

$$
h=\left(\begin{array}{c}
n_{i} \\
i
\end{array}\right)+\left(\begin{array}{c}
n_{i-1} \\
i-1
\end{array}\right)+\cdots+\left(\begin{array}{c}
n_{j} \\
j
\end{array}\right),
$$

where $n_{i}>n_{i-1}>\cdots>n_{j} \geq j \geq 1$. We put $0^{<i>}=0$ and

$$
h^{<i>}=\left(\begin{array}{c}
n_{i}+1 \\
i+1
\end{array}\right)+\left(\begin{array}{c}
n_{i-1}+1 \\
i
\end{array}\right)+\cdots+\left(\begin{array}{c}
n_{j}+1 \\
j+1
\end{array}\right) .
$$

A finite or infinite sequence $\underline{H}=\left(h_{0}, h_{1}, \ldots\right)$ of nonnegative integers is called an $O$-sequence if $\underline{H}$ is the Hilbert function of some $k$-algebra, i.e., $h_{0}=1$ and $0<h_{i+1} \leq h_{i}^{\langle i\rangle}$ for $i=1,2, \ldots$ (cf. [7] and [11, Theorem 2.2] for the details). A sequence $\underline{H}=\left(h_{0}, h_{1}, \ldots, h_{s}\right)$ of positive integers is called a

Received by the editors June 20, 1994.

1991 Mathematics Subject Classification. Primary 13C40, 13D40, 13 F99. 
Gorenstein sequence if $\underline{H}$ is the Hilbert function of some Gorenstein Artin $k$ algebra. Furthermore, we say that a sequence $\underline{H}=\left(h_{0}, h_{1}, \ldots, h_{s}\right)$ of positive integers is unimodal if $h_{0} \leq h_{1} \leq \cdots \leq h_{j} \geq h_{j+1} \geq \cdots \geq h_{s}$.

R. Stanley and A. Iarrobino independently conjectured the following (cf. [11, page 68]).

A sequence $\underline{H}=\left(h_{0}, h_{1}, \ldots, h_{s}\right)$ is a Gorenstein sequence if and only if $\underline{H}$ is a sequence which satisfies the following three conditions:

(i) $\underline{H}$ is unimodal.

(ii) $\underline{H}$ is symmetric, i.e., $h_{s-i}=h_{i}$ for all $i=0,1, \ldots,[s / 2]$.

(iii) $\left(h_{0}, h_{1}-h_{0}, h_{2}-h_{1}, \ldots, h_{t}-h_{t-1}\right)$ is an O-sequence, where $t=\min \{i \mid$ $\left.h_{i} \geq h_{i+1}\right\}$.

A sequence which satisfies the three conditions above is called an SI-sequence.

R. Stanley [11, Theorem 4.1 and Theorem 4.2] showed that all Gorenstein sequences satisfy condition (ii), and that this conjecture is true if $h_{1} \leq 3$. But every Gorenstein sequence does not necessarily satisfy condition (i). The first counterexample was given by R. Stanley [11, Example 4.3], with $\underline{H}=$ $(1,13,12,13,1)$, and others have brought this down to $h_{1}=5$ (cf. [1, Theorem 1] and [3, Example 5.5]). The author does not know whether there exist nonunimodal Gorenstein sequences with $h_{1}=4$.

In this paper, we examine Hilbert functions of Gorenstein Artin $k$-algebras with the following property.

Definition 1.1 (cf. [13, Definition 3.1]). Let $A=\bigoplus_{i=0}^{s} A_{i}$ be an Artin algebra, where $A_{s} \neq 0$. We say that $A$ has the weak Stanley property (WSP for short) if $A$ satisfies the following two conditions:

(i) The Hilbert function of $A$ is unimodal.

(ii) There exists $g \in A_{1}$ such that the $k$-vector space homomorphism $g$ : $A_{i} \longrightarrow A_{i+1}$ defined by $f \longmapsto g f$ is either injective or surjective for all $i=0,1, \ldots, s-1$.

In this case, we say that the pair $(A, g)$ has the WSP.

The main theorem of this paper is the following.

Theorem 1.2. Let $\underline{H}=\left(h_{0}, h_{1}, \ldots, h_{s}\right)$ be a sequence of positive integers. Then $\underline{H}$ is the Hilbert function of some Gorenstein Artin $k$-algebra with the WSP if and only if $\underline{H}$ is an SI-sequence.

This characterization, in characteristic zero, is a special case of the $g$-Theorem by L. J. Billera and C. W. Lee [2] and R. Stanley [12]. The g-Theorem says that an $h$-vector is the $h$-vector of a simplicial polytope if and only if it is an SI-sequence. We give another proof which avoids the hard results of toric varieties and topology used by R. Stanley [12]. That is, the idea for proving this characterization is to use the construction in [5, Corollary 2.5] and [6, Theorem 4.1], a result of linkage [8, Remarque 1.4] and the technique of the proof of [13, Theorem 3.8].

Finally I would like to express my sincere gratitude to the referee for useful advice. 


\section{Preliminaries}

Throughout this paper, let $k$ be an algebraically closed field and let $R=$ $k\left[x_{0}, x_{1}, \ldots, x_{n}\right]$ be the homogeneous coordinate ring of $\mathbf{P}^{n}=\mathbf{P}_{k}^{n}$. Let $X$ be a finite set of points in $\mathbf{P}^{n}$ and let $I(X) \subset R$ be the homogeneous ideal of $X$. The Hilbert function of $X$ is defined by $H(X, i)=H(R / I(X), i)$ for all $i$. Furthermore we put $\Delta H(X, 0)=1, \Delta H(X, i)=H(X, i)-H(X, i-1)$ for all $i=1,2, \ldots$ and $\Delta H(X, i)=0$ for all $i<0$. We denote by $|X|$ the number of points in $X$.

We recall some basic facts about Hilbert functions of points in $\mathbf{P}^{n}$.

Proposition 2.1. Let $X$ be a finite set of points in $\mathbf{P}^{n}$.

(1) $H(X, i) \leq H(X, i+1)$ for all $i \geq 0$.

(2) $H(X, i)=H(X, i+1) \Rightarrow H(X, i+2)=H(X, i+1)$.

(3) $H(X, i)=|X|$ for all $i>>0$.

Put $\sigma(X)=\min \{i \mid \Delta H(X, i)=0\}$.

(4) If $Y \subset X$, then $\sigma(Y) \leq \sigma(X)$.

(5) $H(X, i)=|X|$ for all $i \geq \sigma(X)-1$.

(6) $|X| \geq 2 \Longleftrightarrow \sigma(X) \geq 2$.

(7) $(\Delta H(X, 0), \ldots, \Delta H(X, \sigma(X)-1))$ is an O-sequence.

Proof. The assertions of (1), (2), (3) and (4) follow from [9, Proposition 1 and Proposition 2].

(5) follows from (2) and (3).

(6) Assume that $|X| \geq 2$. Then it follows from (1), (2), (3) and $H(X, 0)=1$ that $H(X, 1) \geq 2$. Hence $\Delta H(X, 1) \geq 1$. Thus $\sigma(X) \geq 2$. Conversely assume that $\sigma(X) \geq 2$. Then since $\Delta H(X, 1) \geq 1$, it follows that $H(X, 1) \geq 2$. Hence $|X| \geq 2$.

(7) follows from [6, Proposition 2.7].

\section{A CONSTRUCTION OF A NUMBER OF GoRENSTEIN ARTIN $k$-ALGEBRAS WITH THE WSP}

In this section, we prove the following four lemmas, which are the key to the proof of Theorem 1.2. That is, for two given geometrically linked sets of points, we give a construction of a number of Gorenstein Artin $k$-algebras with the WSP whose Hilbert functions can be recovered from the Hilbert function of one of the sets (cf. [8] for the details about geometric linkage).

Lemma 3.1. Let $X$ and $Y$ be two finite sets of points in $\mathbf{P}^{n}$ such that $X \cap Y=\varnothing$ and $X \cup Y$ is complete intersection, and put $A=R / I(X)+I(Y)$. Furthermore put $a=\sigma(X)-1, b=\sigma(X \cup Y)-\sigma(X)-1, c=\sigma(X \cup Y)-1$. Assume that $2 \sigma(X) \leq \sigma(X \cup Y)$ and $|X| \geq 2$. Then $H(A)$ is a Gorenstein SI-sequence as follows.

$$
H(A, i)= \begin{cases}H(X, i), & i=0,1, \ldots, a-1, \\ |X|, & i=a, \ldots, b, \\ H(X, c-1-i), & i=b+1, \ldots, c-1,\end{cases}
$$

i.e., $H(A)=\left(1, h_{1}, \ldots, h_{a-1},|X|, \ldots,|X|, h_{a-1}, \ldots, h_{1}, 1\right)$, where $h_{i}=$ $H(X, i)$, and we have $\sigma(A)=c$. 
Proof. By the assumption $2 \sigma(X) \leq \sigma(X \cup Y)$ and $|X| \geq 2$, it is easy to show that $a \leq b, b+1 \leq c-1$ and $a \leq[(c-1) / 2] \leq b$.

First we show that

$$
H(A, i)=H(X, i)+H(X, c-1-i)-|X| \text { for all } i=0,1, \ldots, c .
$$

From [4, Theorem 3 (b)], it follows that

$$
\Delta H(X \cup Y, i)=\Delta H(X, c-i)+\Delta H(Y, i) \text { for all } i=0,1, \ldots, c .
$$

Hence for every $j \geq 0$,

$$
\sum_{i=0}^{j} \Delta H(X \cup Y, i)=\sum_{i=0}^{j} \Delta H(X, c-i)+\sum_{i=0}^{j} \Delta H(Y, i) .
$$

Therefore

$$
\begin{aligned}
\sum_{i=0}^{j} \Delta H(X, c-i) & =\sum_{i=0}^{j} \Delta H(X \cup Y, i)-\sum_{i=0}^{j} \Delta H(Y, i) \\
& =H(X \cup Y, j)-H(Y, j) .
\end{aligned}
$$

Furthermore, from $a \leq c$,

$$
\sum_{i=0}^{c} \Delta H(X, i)=H(X, c)=|X| .
$$

Thus for all $j=0,1, \ldots, c$,

$$
\begin{aligned}
\sum_{i=0}^{j} \Delta H(X, c-i) & =\sum_{i=0}^{c} \Delta H(X, i)-\sum_{i=0}^{c-1-j} \Delta H(X, i) \\
& =|X|-H(X, c-1-j) .
\end{aligned}
$$

Therefore we obtain from (3.1.3) and (3.1.4) that (3.1.5)

$H(X \cup Y, j)-H(Y, j)=|X|-H(X, c-1-j)$ for all $j=0,1, \ldots, c$.

On the other hand, from the following exact sequence

$$
0 \rightarrow R / I(X \cup Y) \rightarrow R / I(X) \oplus R / I(Y) \rightarrow A \rightarrow 0,
$$

we have

$$
\begin{aligned}
H(A, i) & =H(R / I(X), i)+H(R / I(Y), i)-H(R / I(X \cup Y), i) \\
& =H(X, i)+H(Y, i)-H(X \cup Y, i) .
\end{aligned}
$$

Hence we obtain from (3.1.5) that

$$
H(A, i)=H(X, i)+H(X, c-1-i)-|X| .
$$

Next we show that $\sigma(A)=c$. Namely, it is enough to show that

$$
H(A, c-1) \neq 0 \text { and } H(A, c)=0 .
$$

Since $a \leq b$ and $b+1 \leq c-1$, we have $a<c-1$. Hence $H(X, c-1)=|X|$. 
Therefore when $i=c-1$, it follows from (3.1.2) that

$$
\begin{aligned}
H(A, c-1) & =H(X, c-1)+H(X, 0)-|X| \\
& =|X|+1-|X|=1 .
\end{aligned}
$$

Furthermore when $i=c$, we obtain

$$
\begin{aligned}
H(A, c) & =H(X, c)+H(X,-1)-|X| \\
& =|X|+0-|X|=0 .
\end{aligned}
$$

We show equality (3.1.1). Since $a+b=\sigma(X)-1+\sigma(X \cup Y)-\sigma(X)-1=c-1$, we have $a \leq c-1-i$ for all $i=0,1, \ldots, b$. Hence

$$
H(X, c-1-i)=|X| \text { for all } i=0,1, \ldots, b .
$$

On the other hand we obtain from $a<b+1$ that

$$
H(X, i)=|X| \text { for all } i=b+1, \ldots
$$

Hence, from (3.1.2), we get

$$
H(A, i)= \begin{cases}H(X, i) & \text { for all } i=0,1, \ldots, b \\ H(X, c-1-i) & \text { for all } i=b+1, \ldots, c-1 .\end{cases}
$$

Thus equality (3.1.1) holds by noting $H(X, i)=|X|$ for all $i=a, \ldots, b$. Hence from Proposition $2.1(1)$ and (7), $H(A)$ is an SI-sequence. Furthermore by [8, Remarque 1.4], $A$ is a Gorenstein Artin $k$-algebra. Hence $H(A)$ is a Gorenstein sequence.

Lemma 3.2. With the same notation as in Lemma 3.1, let $L \subset \mathbf{P}^{n}$ be a hyperplane defined by a polynomial $G \in R_{1}$, and let $g \in A_{1}$ be the image of $G$. Assume that $2 \sigma(X) \leq \sigma(X \cup Y)$ and $X \cap L=\varnothing$. Then $(A, g)$ has the WSP.

Proof. It is enough to show that $g: A_{i} \longrightarrow A_{i+1}$ is either injective or surjective for all $i$. Put $B=R / I(X)=\bigoplus_{i \geq 0} B_{i}$, and let $\bar{G} \in B_{1}$ be the image of $G$. Consider the following commutative diagram:

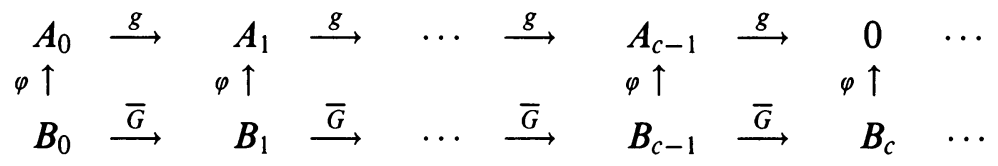

where $\varphi$ is the canonical homomorphism $B \longrightarrow A$. It follows immediately from $X \cap L=\varnothing$ that $\bar{G}$ is not a zero-divisor in $B$. Hence $\bar{G}: B_{i} \longrightarrow$ $B_{i+1}$ is injective for all $i$. Furthermore we have from Proposition 2.1 (5) that $H(B, i)=|X|$ for all $i \geq a$. Therefore $\bar{G}: B_{i} \longrightarrow B_{i+1}$ is bijective for all $i \geq a$. We obtain from (3.1.6) that $H(A, i)=H(B, i)$ for all $i=0,1, \ldots, b$. Hence since the homogeneous part of the canonical homomorphism $\varphi: B \longrightarrow A$ is surjective, $\varphi: B_{i} \longrightarrow A_{i}$ is bijective for all $i=0,1, \ldots, b$. Thus it follows immediately that

$$
g: A_{i} \longrightarrow A_{i+1} \text { is } \begin{cases}\text { injective } & \text { for all } i=0,1, \ldots, a-1 \\ \text { bijective } & \text { for all } i=a, \ldots, b-1 \\ \text { surjective } & \text { for all } i=b, \ldots, c-1\end{cases}
$$


Lemma 3.3. With the same notation as in Lemma 3.2, let $d$ be an integer such that $1 \leq d \leq \sigma(X \cup Y)-2 \sigma(X)$ and let $\left(0: g^{d}\right)$ denote the homogeneous ideal generated by homogeneous elements $f \in A$ such that $g^{d} f=0$. Then $H\left(A /\left(0: g^{d}\right)\right)$ is a Gorenstein SI-sequence as follows.

$$
H\left(A /\left(0: g^{d}\right), i\right)= \begin{cases}H(X, i), & i=0,1, \ldots, a-1, \\ |X|, & i=a, \ldots, b-d, \\ H(X, c-1-i-d), & i=b+1-d, \ldots, c-1-d,\end{cases}
$$

and $\sigma\left(A /\left(0: g^{d}\right)\right)=c-d$.

Proof. Put $\bar{A}=A /\left(0: g^{d}\right)$. Note that the $i$-th graded piece of $\bar{A}$ is

$$
A_{i} / \operatorname{ker}\left[g^{d}: A_{i} \longrightarrow A_{i+d}\right] \text {. }
$$

Since $1 \leq d \leq \sigma(X \cup Y)-2 \sigma(X)$, obviously $a \leq b-d$. Hence we obtain from (3.2.1) that

$$
g^{d}: A_{i} \longrightarrow A_{i+d} \text { is } \begin{cases}\text { injective, } & i=0,1, \ldots, b-d, \\ \text { surjective, } & i=b+1-d, \ldots\end{cases}
$$

Thus we get the following identification

$$
\bar{A} \cong A_{0} \oplus A_{1} \oplus \cdots \oplus A_{b-d} \oplus A_{b+1} \oplus A_{b+2} \oplus \cdots \oplus A_{c-1} \oplus 0 \oplus \cdots .
$$

Obviously $\sigma(\bar{A})=c-d$ and

$$
H(\bar{A}, i)= \begin{cases}H(A, i), & i=0,1, \ldots, b-d, \\ H(A, i+d), & i=b+1-d, \ldots, c-1-d .\end{cases}
$$

Hence, from (3.1.6), we obtain equality (3.3.1). Thus $H(\bar{A})$ is an SI-sequence.

Next we show that $H(\bar{A})$ is a Gorenstein sequence, i.e., $\bar{A}$ is Gorenstein. Put $\operatorname{Soc}(\bar{A})=\left\{\bar{y} \in \bar{A} \mid \bar{A}_{1} \bar{y}=(0)\right\}$, where $\bar{y}$ is the image of $y \in A$. We note that $\sigma(\bar{A})=c-d$ and $\operatorname{dim}_{k}(\bar{A})_{c-1-d}=1$. It is enough to show that $\operatorname{Soc}(\bar{A})=(\bar{A})_{c-1-d}$. Let $\bar{y} \in \bar{A}_{i}\left(y \in A_{i}, i<c-1-d\right)$ be an element such that $\bar{y} \in \operatorname{Soc}(\bar{A})$. Hence $A_{1} y \subset\left(0: g^{d}\right)$. Since $\operatorname{Soc}(A)=A_{c-1}$, we have $y g^{d} \in A_{c-1}$. On the other hand, $y g^{d} \in A_{i+d}$. Since $i+d<c-1$, we obtain $y g^{d}=0$. Hence $\bar{y}=0$. Thus $\operatorname{Soc}(\bar{A})=(\bar{A})_{c-1-d}$.

Lemma 3.4. With the same notation as in Lemma 3.3, let $\bar{g}$ be the image of $g$ in $A /\left(0: g^{d}\right)$. Then $\left(A /\left(0: g^{d}\right), \bar{g}\right)$ is a Gorenstein Artin $k$-algebra with the WSP.

Proof. It is enough to show that $\bar{g}: \bar{A}_{i} \longrightarrow \bar{A}_{i+d}$ is either injective or surjective for all $i$. It is easy to show that, with the identification (3.3.2), the multiplication $\bar{g}: \bar{A} \longrightarrow \bar{A}$ is described as

$$
A_{0} \stackrel{g}{\longrightarrow} A_{1} \stackrel{g}{\longrightarrow} \cdots \stackrel{g}{\longrightarrow} A_{b-d} \stackrel{g^{d+1}}{\longrightarrow} A_{b+1} \stackrel{g}{\longrightarrow} A_{b+2} \stackrel{g}{\longrightarrow} \cdots \stackrel{g}{\longrightarrow} A_{c-1} .
$$

The only part which is not clear is $A_{b-d} \stackrel{g^{d+1}}{\longrightarrow} A_{b+1}$. But we obtain from $b-d \geq a$ and (3.2.1) that $A_{b-d} \stackrel{g^{d+1}}{\longrightarrow} A_{b+1}$ is surjective.

Remark 3.5. Let $X$ be a finite set of points in $\mathbf{P}^{n}$ and let $j$ be an integer. Then it is easy to construct a finite set $Y$ of points in $\mathbf{P}^{n}$ such that $X \cap Y=\varnothing$, 
$X \cup Y$ is complete intersection and $\sigma(X \cup Y) \geq j$. For example we construct $Y$ as follows. We may assume that $X \cap L=\varnothing$, where $L$ is the hyperplane defined by the equation $x_{0}=0$. Obviously there exist distinct elements $a_{i, j} \in k$ $(1 \leq i \leq n, 1 \leq j \leq m)$ such that $X \subset Z$, where $Z=\left\{\left[1 ; a_{1, j} ; \ldots ; a_{n, j}\right] \mid\right.$ $1 \leq j \leq m\}$. Then it is easy to show that $Z$ is complete intersection and $\sigma(Z)=n m-(n-1)$. Furthermore for a sufficiently large $m$, we get $\sigma(Z) \geq j$. We put $Y=\{P \in Z \mid P \notin X\}$. Then $Y$ satisfies the conditions above.

\section{Proof of Theorem 1.2}

Before proving Theorem 1.2, we have to recall a result in [5] and [6].

Definition 4.1 (cf. [6, Definition 2.9]). An O-sequence $\underline{b}=\left(b_{0}, b_{1}, \ldots, b_{i}, \ldots\right)$ is called differentiable if its difference $\Delta \underline{b}=\left(b_{0}, b_{1}-b_{0}, \ldots, b_{i}-b_{i-1}, \ldots\right)$ is also an O-sequence.

Proposition 4.2 (cf. [5, Corollary 2.5] and [6, Theorem 4.1]). Let $\underline{b}$ be a differentiable $O$-sequence. Then there is a reduced $k$-algebra over an infinite field $k$ with Hilbert function $\underline{b}$.

We now start to prove Theorem 1.2.

Proof of Theorem 1.2. Assume that $\underline{H}$ is the Hilbert function of some Gorenstein Artin $k$-algebra $(A, g)$ with the WSP. Then the Hilbert function of $A / g A$ is the sequence $\left(h_{0}, h_{1}-h_{0}, h_{2}-h_{1}, \ldots, h_{t}-h_{t-1}\right)$, where $h_{i}=H(A, i)$ and $t=\min \{i \mid H(A, i)(A, i+1)\}$. Hence $\underline{H}$ is an SI-sequence.

Conversely assume that $\underline{H}$ is an SI-sequence.

If $h_{1}=1$, then it is easy to show that $h_{i}=1$ for all $i=0,1, \ldots, s$. Hence $\underline{H}$ is the Hilbert function of $A=k\left[x_{0}\right] /\left(x_{0}^{s+1}\right)$, which is a Gorenstein Artin $k$-algebra with the WSP.

Let $h_{1} \geq 2$. We put $t=\min \left\{i \mid h_{i} \geq h_{i+1}\right\}$. Hence

$$
\underline{H}=\left(1, h_{1}, \ldots, h_{t-1}, h_{t}, \ldots, h_{t}, h_{t-1}, \ldots, h_{1}, 1\right) .
$$

We consider the infinite sequence $\underline{b}=\left\{b_{i}\right\}_{i \geq 0}$, where $b_{i}=h_{i}$ for all $i=$ $0,1, \ldots, t$ and $b_{i}=h_{t}$ for all $i=t+1, \ldots$. Since $\underline{H}$ is an SI-sequence, $\underline{b}$ is a differentiable O-sequence. Hence, by Proposition 4.2, there exists a finite set $X$ of points in $\mathbf{P}^{n}$ such that $H(X)=\underline{b}$, where $n=h_{1}-1$. We may assume that $X \cap L=\varnothing$, where $L$ is the hyperplane defined by the equation $x_{0}=0$. Furthermore by Remark 3.5, there exists a finite set $Y$ of points in $\mathbf{P}^{n}$ such that $X \cap Y=\varnothing, X \cup Y$ is complete intersection and $\sigma(X \cup Y) \geq s+2$. Since $2 t \leq s$ and $\sigma(X)=t+1$, it follows that $2 \sigma(X) \leq s+2$. Hence $2 \sigma(X) \leq \sigma(X \cup Y)$. Put $A=R / I(X)+I(Y)$ and $d=\sigma(X \cup Y)-s-2$, and let $g$ be the image of $x_{0}$ in $A$. Note that $d \geq 0$. Furthermore, we put $a=\sigma(X)-1, b=\sigma(X \cup Y)-\sigma(X)-1$ and $c=\sigma(X \cup Y)-1$. Then we obtain by Lemma 3.1 and Lemma 3.3 that

$$
H\left(A /\left(0: g^{d}\right), i\right)= \begin{cases}H(X, i), & i=0,1, \ldots, a-1, \\ |X|, & i=a, \ldots, b-d, \\ H(X, c-1-i-d), & i=b+1-d, \ldots, c-1-d,\end{cases}
$$

where $g^{0}=1$. It is easy to show that $a \leq[s / 2] \leq b-d$. Hence

$$
H\left(A /\left(0: g^{d}\right), i\right)=H(X, i)=b_{i}=h_{i}
$$


for all $i=0,1, \ldots,[s / 2]$. Thus since $H\left(A /\left(0: g^{d}\right)\right)$ is symmetric and $c-$ $1-d=s$, it follows immediately that $H\left(A /\left(0: g^{d}\right)\right)=\underline{H}$. Furthermore by Lemma 3.2 and Lemma $3.4, A /\left(0: g^{d}\right)$ is a Gorenstein Artin $k$-algebra with the WSP. This completes the proof of Theorem 1.2.

\section{REFERENCES}

1. D. Bernstein and A. Iarrobino, A nonunimodal graded Gorenstein Artin algebra in codimension five, Comm. Algebra 20 (1992), 2323-2336.

2. L. J. Billera and C. W. Lee, Sufficiency of McMullen's conditions for $f$-vectors of simplicial polytopes, Bull. Amer. Math. Soc. (N.S.) 2 (1980), 181-185.

3. M. Boij and D. Laksov, Nonunimodality of graded Gorenstein Artin algebras, Proc. Amer. Math. Soc. 120 (1994), 1083-1092.

4. E. D. Davis, A. V. Geramita, and F. Orecchia, Gorenstein algebras and the Cayley-Bacharach theorem, Proc. Amer. Math. Soc. 93 (1985), 593-597.

5. A. V. Geramita, D. Gregory, and L. Roberts, Monomial ideals and points in projective space, J. Pure Appl. Algebra 40 (1986), 33-62.

6. A. V. Geramita, P. Maroscia, and L. Roberts, The Hilbert function of a reduced $k$-algebra, The Curves Seminar at Queen's Vol. II, Queen's Papers in Pure and Appl. Math., vol. 61, Queen's Univ., Kingston, Ontario, 1982.

7. F. S. Macaulay, Some properties of enumeration in the theory of modular systems, Proc. London Math. Soc. (3) 26 (1927), 531-555.

8. C. Peskine and L. Szpiro, Liaison des variétés algébriques. I, Invent. Math. 26 (1974), 271-302.

9. A. Sodhi, On the intersecticn of a hypersurface with a finite set of points in $\mathbf{P}^{n}$, J. Pure Appl. Algebra 74 (1991), 85-94.

10. R. Stanley, Cohen-Macaulay rings and constructible polytopes, Bull. Amer. Math. Soc. 81 (1975), 133-135.

11. _ Hilbert functions of graded algebras, Adv. in Math. 28 (1978), 57-83.

12. _ The number of faces of a simplicial convex polytope, Adv. in Math. 35 (1980), 236-238.

13. J. Watanabe, The Dilworth number of Artinian rings and finite posets with rank function, Commutative Algebra and Combinatorics, Adv. Stud. Pure Math., vol. 11, Academic Press, Boston, MA, 1987, pp. 303-312.

Department of Management and Information Science, Shikoku University, Furukawa OHJIN-CHO,TOKUSHIMA 771-11, JAPAN

E-mail address: harima@keiei.shikoku-u.ac.jp 